Jpn. J. Infect. Dis., 74, 325-332, 2021

Original Article

\title{
Reverse Transcription Loop-Mediated Isothermal Amplification Assay-Based Infection Control Strategies for COVID-19 in a Hospital Under the State of Emergency in Tokyo, Japan in Spring 2020
}

\author{
Muneyoshi Kimura ${ }^{1,2 *}$, Masaru Baba ${ }^{1,2}$, Shunichiro Maruyama ${ }^{2,3}$, Sho Ogura ${ }^{1,2}$, Ryosuke Yamamuro $^{1,2}$, \\ Takashi Sakoh $^{1,2}$, Toshiyuki Kishida ${ }^{1,2}$, Yumi Nagamine ${ }^{2,4}$, Yusuke Endo ${ }^{1}$, Chikako Okada ${ }^{1,2}$, \\ Namiko Takahashi, ${ }^{2,3}$, and Hideki Araoka ${ }^{1,2,5}$ \\ ${ }^{1}$ Department of Infectious Diseases, ${ }^{2}$ Department of Infection Control and Prevention, \\ ${ }^{3}$ Nursing Department, and ${ }^{4}$ Department of Pharmacy, Toranomon Hospital, Tokyo; \\ and ${ }^{5}$ Okinaka Memorial Institute for Medical Research, Tokyo, Japan
}

\begin{abstract}
SUMMARY: Studies describing reverse transcription loop-mediated isothermal amplification (RTLAMP) assay-based infection control strategies (LAMP-based ICSs) for coronavirus disease 2019 (COVID-19) are limited. We reviewed the medical records of cases in which RT-LAMP was performed. Standard ICSs and LAMP-based ICSs were implemented during the study period. The strategies were intended to impose longer periods of infection control precautions (ICPs) for specific patients, such as those with a history of exposure to COVID-19 patients and/or bilateral ground glass opacities (bGGO) on chest computed tomography (CT). Of 212 patients, which included 13 confirmed COVID-19 patients in the diagnostic cohort, exposure to COVID-19 patients $(P<0.0001)$ and chest CT bGGO $(P$ $=0.0022)$ were identified as significant predictors of COVID-19. In the 173 hospitalized patients in which the results of the first RT-LAMP were negative, the duration of ICPs was significantly longer in patients with exposure to COVID-19 and/or a high clinical index of suspicion and patients with bGGO than in the remaining patients $(P=0.00046$ and $P=0.0067$, respectively). Additionally, no confirmed COVID-19 cases indicating nosocomial spread occurred during the study period. Establishing a comprehensive system that combines rational LAMP-based ICSs with standard ICSs might be useful for preventing nosocomial spread.
\end{abstract}

\section{INTRODUCTION}

Coronavirus disease 2019 (COVID-19) is an emerging infectious disease caused by severe acute respiratory syndrome coronavirus 2 (SARS-CoV-2). It has rapidly spread worldwide through direct personto-person transmission. The spread of infection is thought to occur through close-range contact, mainly via respiratory droplets (1). In general, it is difficult to distinguish COVID-19 from other respiratory infections based on clinical symptoms alone, because the symptoms of COVID-19 are nonspecific (2). This allowed COVID-19 to go unnoticed in the early phase and spread rapidly. In this context, both an appropriate diagnostic strategy and infection control precautions

Received September 12, 2020. Accepted December 1, 2020. J-STAGE Advance Publication December 25, 2020.

DOI: 10.7883 /yoken.JJID.2020.808

*Corresponding author: Mailing address: Department of Infectious Diseases, Toranomon Hospital, 2-2-2 Toranomon, Minato-ku, Tokyo 105-8470, Japan. Tel:+81-3-3588-1111, Fax:+81-3-3582-7068, E-mail: muneyoshi-k@toranomon. gr.jp
(ICPs) are indispensable to prevent nosocomial spread. According to guidelines, nucleic acid amplification testing (NAAT), such as reverse-transcription polymerase chain reaction (RT-PCR), is recommended for diagnosing COVID-19 $(3,4)$.

The loop-mediated isothermal amplification (LAMP) assay developed in Japan is a type of NAAT (5). LAMP is simple, easy to perform, and cost effective and amplifies DNA with high specificity and efficiency under isothermal conditions $\left(60-65^{\circ} \mathrm{C}\right)(5)$. However, few clinical studies have reported the utility of reverse transcription LAMP (RT-LAMP) in clinical settings for COVID-19. In previous studies, RT-LAMP showed an accuracy comparable to that of RT-PCR for detecting SARS-CoV-2 in clinical specimens $(6,7)$. However, false-negative RT-PCR results were reported in a previous study (8). Thus, COVID-19 should not be ruled out based on RT-PCR (8) or RT-LAMP alone, and clinicians should consider the clinical course when deciding whether to discontinue ICPs for the patient whenever COVID-19 is included in the differential diagnosis, even if NAAT is negative (8). Guidance has been issued on when to discontinue ICPs for confirmed COVID-19 patients (9). Meanwhile, few reports 


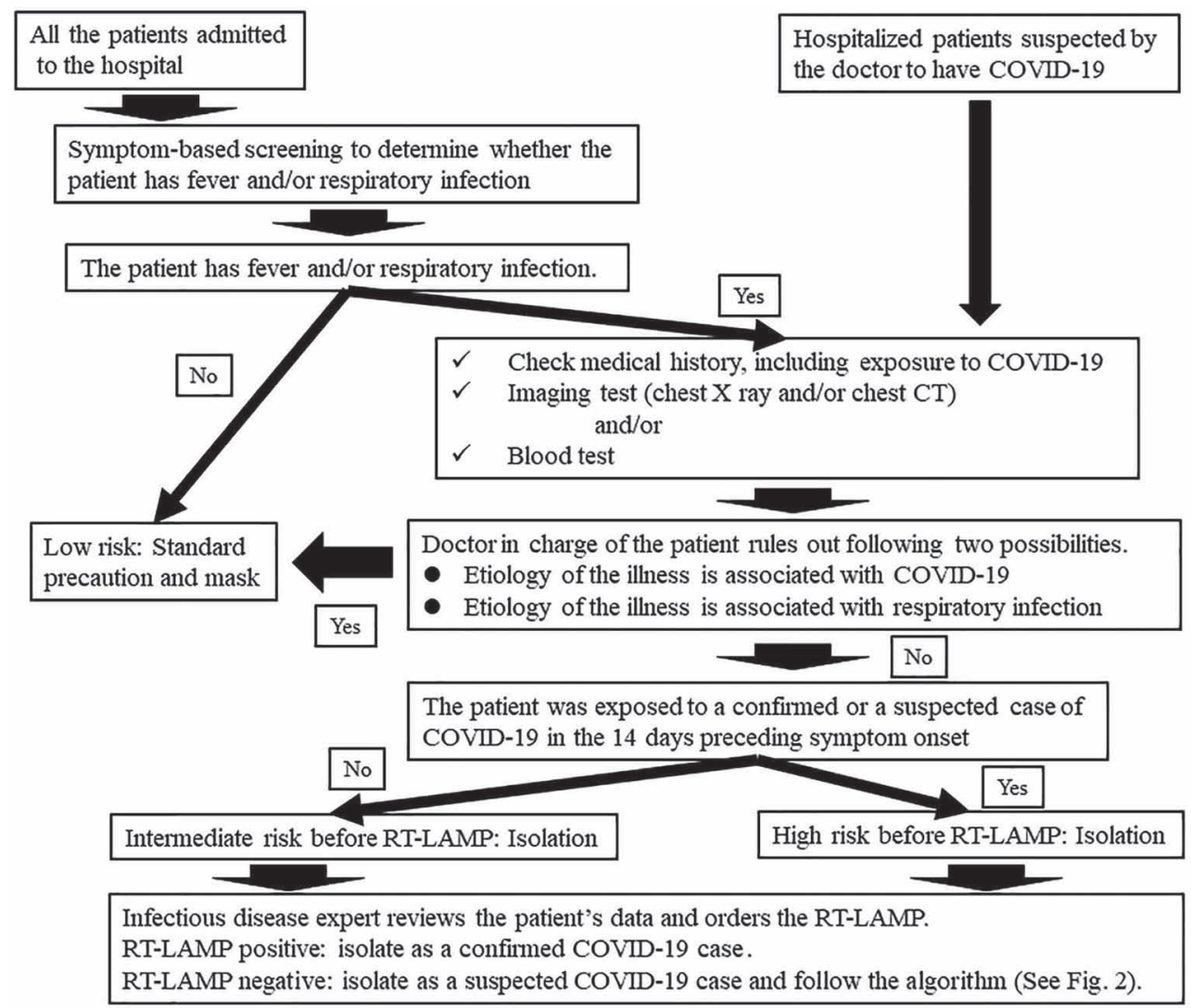

Fig.1. Risk assessment strategy for the patients admitted to the hospital and suspected of having possible nosocomial onset of COVID-19 before the first RT-LAMP. If patients categorized as low risk had a history of exposure to COVID-19, they were individually quarantined in a single room of the hospital for at least 14 days or their admission was postponed for at least 14 days.

have investigated the removal of ICPs for suspected COVID-19 patients with negative NAAT results, although strategies to resolve these issues are needed in the real-world setting. Therefore, the aim of this study was to provide real-world data on RT-LAMP-based infection control strategies implemented during the state of emergency in Tokyo in spring 2020.

\section{MATERIALS AND METHODS}

A retrospective analysis of patients aged $\geq 20$ years who underwent RT-LAMP assay for detection of SARSCoV-2 between April 1 and May 25, 2020 at Toranomon Hospital (820 beds; Tokyo, Japan) was performed. The medical and virology records of the patients during the study period were reviewed. We also observed all the staff and all hospitalized patients of Toranomon Hospital during the study period and the subsequent 14-day observational period (until June 9, 2020) to evaluate nosocomial transmission of SARS-CoV-2. This study was approved by the Institutional Review Bord of Toranomon Hospital.

Definition: A confirmed COVID-19 patient was defined as a patient with $\geq 1$ positive RT-LAMP and/or RT-PCR results for detecting SARS-CoV-2. A history of exposure was defined as any history of close contact with a confirmed COVID-19 patient within 14 days preceding symptom onset. Close contact was defined as described in a previous study (10). ICPs were such that patients were isolated and received medical care provided by healthcare workers wearing appropriate personal protective equipment (PPE). ICP duration was defined as the number of days between the start of ICPs and the day of discontinuation. Healthcare workers with potential exposure to COVID-19 patients were categorized into low-, intermediate-, or high-risk groups based on guidance $(11,12)$. Only data for chest computed tomography (CT) scans taken $72 \mathrm{~h}$ before the first RT-LAMP and on the day of the first RT-LAMP were analyzed in this study.

Standard infection control strategies against COVID-19 during the study period: Standard infection control strategies were implemented during the study period. Most implementations comply with international guidelines $(1,13)$. The details are as follows. During the study period, all staff, inpatients, outpatients, and visitors at our institution wore masks. In addition, the infection control team strongly recommended strict hand hygiene, which was in line with the WHO guideline (14). Furthermore, standard environmental disinfection and educational programs for healthcare workers were provided (e.g., lectures on how to handle PPE and implement zoning in the ward for confirmed COVID-19 patients).

Risk assessment system for patients with possible COVID-19 during the study period: Risk assessment was performed using specific algorithms (Figs. 1 and 2) for all patients admitted to our hospital and for hospitalized patients who were suspected to have COVID-19 by their attending doctors. The algorithms 
The ID expert's screening by using electronic medical chart: the patient fulfill at least one of the followings.

$\checkmark$ The patient exposed to a confirmed COVID-19 case within 14 days prior to the onset of symptoms.

$\checkmark$ The patient exposed to a suspected COVID-19 case who is waiting for the NAAT result within 14 days prior to the onset of symptoms.

$\checkmark$ The ID doctor strongly suspects the patient has COVID-19 because of clinical course and imaging test of the patient.
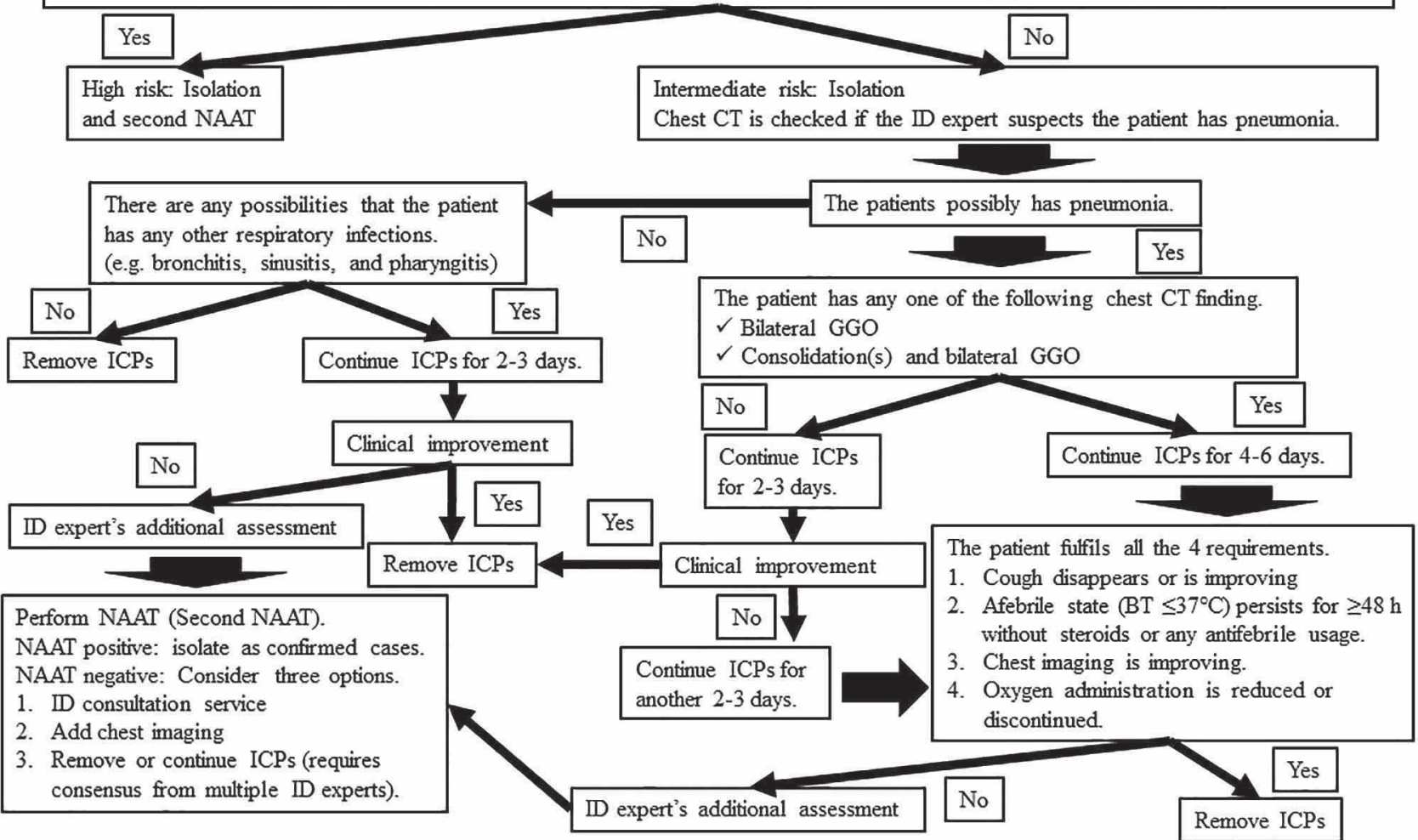

Fig.2. Risk assessment strategy and requirements to discontinue the infection control precautions for whom the first RT-LAMP result was negative. If a second NAAT was performed, the ID expert could select RT-LAMP or RT-PCR. The ID expert evaluated patients who were deemed intermediate- and high-risk once per weekday to decide whether they fulfilled the requirements for discontinuation. If the ID expert had a high index of suspicion of possible COVID-19, they could schedule the second NAAT earlier than the time recommended by the algorithm. When an alternative definitive diagnosis was made, the ID expert discontinued the infection control precautions even when the patient did not fulfill the requirements. The infection control precautions required that the patient be isolated and receive medical care from health care workers dressed in appropriate personal protective equipment. Clinical improvement meant that the patients fulfilled all of the following requirements: i) cough disappears and improves, ii) afebrile state $\left(\mathrm{BT} \leq 37^{\circ} \mathrm{C}\right.$ ) persists for $\geq 24 \mathrm{~h}$ without use of steroids or antipyretic medicine, iii) chest imaging improves (if the patient has pneumonia), and iv) oxygen administration is reduced or discontinued.

were established at the beginning of April 2020. First, patients were divided into 1 of the 3 risk categories (low, intermediate, or high) before receiving RT-LAMP. The evaluation was performed by the doctor responsible for the patient's care. Next, infectious disease (ID) experts ordered an RT-LAMP assay for patients categorized as either intermediate or high risk before the test (Fig. 1). Nasopharyngeal specimens obtained from welltrained nurses wearing appropriate PPE were used in the assay. Then, confirmed COVID-19 patients (those with positive RT-LAMP results) were isolated in a negative pressure ward for care of only confirmed patients. Patients were managed by a COVID-19 team. Patients whose RT-LAMP results were negative were divided into 1 of 2 risk categories, either intermediate or high risk, according to the algorithm (Fig. 2). The patient's clinical information required to perform the risk assessment and operate the algorithm was obtained mainly from a medical chart review by the ID expert. Furthermore, these patients were isolated in a private room. If they had at least 1 positive NAAT result, they were transferred to the negative-pressure ward. The high-risk patients whose second NAAT result was negative were each isolated in a private room for at least 10 days unless a definite alternative diagnosis was made or the patient died.

Concerning intermediate-risk patients whose first RT-LAMP results were negative, the ID expert decided when to discontinue the ICPs by referring to the algorithm (Fig. 2).

Health care workers who cared for high-risk patients, intermediate-risk patients, and/or confirmed COVID-19 patients wore appropriate PPE, as recommended by our institute's guidelines (Table 1).

Other infection control implementation: Eating and drinking face-to-face with friends and colleagues were prohibited in and outside the hospital. In addition, the rooms or venues for patient socialization were closed at our institution.

RT-LAMP assay: Viral RNA was extracted from nasopharyngeal specimens using an influenza virus extraction reagent (Eiken Chemical, Tokyo, Japan). The 
Table 1. Recommended personal protective equipment for each risk category in Toranomon Hospital

\begin{tabular}{ccccccc}
\hline Risk category & Medical mask & N95 mask & Eye protection & Gown & Glove & Medical cap \\
\hline Low risk & + & - & \pm & - & - & - \\
Intermediate risk & + & \pm & \pm & + & + & + \\
High risk & - & + & + & + & + & + \\
\hline
\end{tabular}

Algorithms for risk stratification are shown in Fig. 1 and Fig. 2.

+ , Recommended; \pm , Recommended only during aerosol-generating procedures; -, Not recommended.

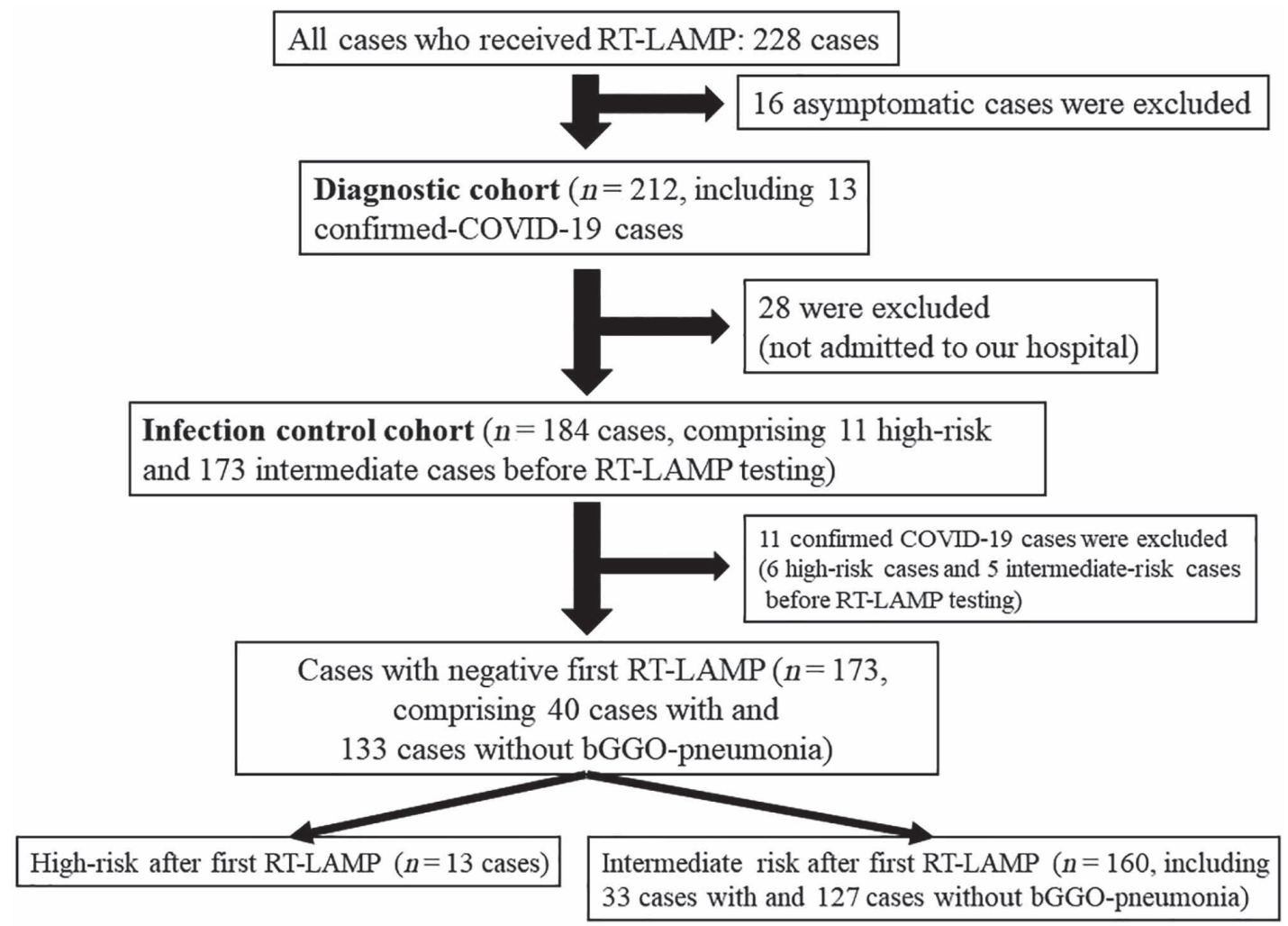

Fig. 3. Flow chart of patient assignment to each cohort and group. bGGO, bilateral-ground glass opacities.

method of amplification of SARS-CoV-2 RNA by RTLAMP in our hospital was as previously described (7). At our institution, the RT-LAMP assay was performed twice a day on weekdays only and once daily during consecutive holidays between May 2 and May 6, 2020.

RT-PCR assay: Nasopharyngeal specimens obtained from patients were sent to the BML General Laboratory (Tokyo, Japan). RT-PCR was performed as previously described (15). Some patients who underwent RTLAMP were also tested using RT-PCR as the second NAAT (Fig. 2).

Statistical analysis: Categorical variables were compared using Fisher's exact test. The Mann-Whitney $U$ test was performed to test the equality of continuous variables. Variables with a $P$-value of $<0.20$ were entered into logistic regression models with a stepwise selection method for multivariate analysis to identify independent predictors of COVID-19. Significance was set at an $\alpha$ value of 0.05 . All statistical analyses were performed with EZR (Saitama Medical Center, Jichi Medical University), which is a graphical user interface for R (The R Foundation for Statistical Computing, Wien, Austria) (16).

\section{RESULTS}

Overview of patients who underwent RT-LAMP: RT-LAMP was performed for 228 patients between April 1 and May 25, 2020 for the first time. In total, 13 patients were diagnosed with COVID-19 (Fig. 3). All 13 patients were diagnosed with confirmed COVID-19 during the first RT-LAMP testing. There were no patients in whom the first RT-LAMP results were negative and the second NAAT results were positive. Of the 228 patients, 212 had at least 1 symptom and/or clinical manifestation (e.g., abnormality on chest CT), indicating the possibility of COVID-19. Therefore, these 212 patients were included in the diagnostic cohort (Fig. 3). The characteristics of the patients in the diagnostic cohort are shown in Table 2. The remaining 16 patients were asymptomatic when they were tested and had negative RT-LAMP results. These 16 patients were tested due to post-exposure to COVID-19 during screening (7 patients), a requirement for transfer to another institution (5 patients), pre-procedure (e.g., presurgery) screening ( 2 patients), and others ( 2 patients). 
Table 2. The characteristics of the 212 cases in the diagnostic cohort and the analysis of predictors of COVID-19

\begin{tabular}{|c|c|c|c|c|}
\hline \multirow[b]{2}{*}{ Characteristic } & \multicolumn{4}{|c|}{ Result for the following patients } \\
\hline & $\begin{array}{c}\text { Total } \\
(N=212)\end{array}$ & $\begin{array}{l}\text { COVID-19 cases } \\
\qquad(N=13)\end{array}$ & $\begin{array}{l}\text { None COVID-19 cases } \\
\qquad(N=199)\end{array}$ & $P$ value \\
\hline Age, median years (range) & $68(22-97)$ & $78(22-92)$ & $68(23-97)$ & 0.27 \\
\hline \multicolumn{5}{|l|}{ Gender } \\
\hline Male / Female & $109 / 103$ & $4(31) / 9(69)$ & $105(53) / 94(47)$ & 0.16 \\
\hline \multicolumn{5}{|l|}{ Comobidities } \\
\hline Diabetes Melitus & 33 & $2(15)$ & $31(16)$ & 1 \\
\hline Hypertension & 78 & $4(31)$ & $74(37)$ & 0.77 \\
\hline Chronic Heart Failure & 10 & $0(0)$ & $10(5)$ & 1 \\
\hline Chronic Renal Failure & 54 & $1(7.7)$ & $53(27)$ & 0.19 \\
\hline Chronic Renal Failure on hemodialysis & 8 & $1(7.7)$ & $7(3.5)$ & 0.4 \\
\hline Liver Cirrosis & 3 & $0(0)$ & $3(1.5)$ & 1 \\
\hline Hematological Malignancy & 30 & $1(7.7)$ & $29(15)$ & 0.7 \\
\hline Solid tumor & 47 & $0(0)$ & $47(24)$ & 0.077 \\
\hline Bronchial athma & 13 & $1(7.7)$ & $12(6)$ & 0.57 \\
\hline Chronic Obstractive Pulmonary Disease & 7 & $0(0)$ & $7(3.5)$ & 1 \\
\hline Interstitial Lung Disease & 8 & $0(0)$ & $8(4)$ & 1 \\
\hline \multicolumn{5}{|l|}{ Medical status } \\
\hline Allogeneic Hematopoietic Stem Cell Transplantation & 11 & $0(0)$ & $11(5.5)$ & 1 \\
\hline Solid Organ Transplantation & 2 & $0(0)$ & $2(1)$ & 1 \\
\hline \multicolumn{5}{|l|}{ Clinical characteristics } \\
\hline Prior exposure to COVID-19 & 15 & $7(54)$ & $8(4)$ & $<0.00001$ \\
\hline At least one respiratory symptoms & 124 & $11(85)$ & $113(57)$ & 0.078 \\
\hline \multicolumn{5}{|l|}{ Chest $\mathrm{CT}$ imaging and chest $\mathrm{X}$ ray } \\
\hline Pneumonia & 115 & $10(77)$ & $105(53)$ & 0.15 \\
\hline Any bGGO on CT & 51 & $9(69)$ & $42(21)$ & 0.00047 \\
\hline bGGO without consolidation on $\mathrm{CT}$ & 38 & $7(54)$ & $31(16)$ & 0.0027 \\
\hline bGGO with consolidation(s) on $\mathrm{CT}$ & 13 & $2(15)$ & $11(5.5)$ & 0.18 \\
\hline Multi variate analysis & & $\mathrm{aOR}$ & $95 \%$ confidence interval & $P$ value \\
\hline Gender & & NR & NR & NR \\
\hline Chronic renal failure & & NR & NR & NR \\
\hline Solid tumor & & NR & NR & NR \\
\hline Prior exposure to COVID-19 & & 32 & $7.1-147$ & $<0.0001$ \\
\hline At least one respiratory symptom & & NR & NR & NR \\
\hline Pneumonia & & NR & NR & NR \\
\hline Any bGGO on CT & & 9.8 & $2.3-42$ & 0.0022 \\
\hline bGGO without consolidation on CT & & NR & NR & NR \\
\hline bGGO with consolidation(s) on $\mathrm{CT}$ & & NR & NR & NR \\
\hline
\end{tabular}

COVID-19, coronavirus disease 2019; Allo-HSCT, allogeneic hematopoietic stem cell transplantation; bGGO, bilateral-groundglass-opacity; aOR, adjusted odds ratio; NR, not retain final model.

Additionally, in the diagnostic cohort, 113 patients had pneumonia evident on chest $\mathrm{CT}$, and 2 patients had pneumonia evident on chest radiography. Moreover, 184 of the 212 patients in the cohort required admission to our hospital and were included in the infection control cohort (Fig. 3) to be evaluated using the algorithm shown in Fig. 1.

Analysis of the diagnostic cohort: History of prior exposure to COVID-19 patients and bilateral groundglass opacities (bGGO) on chest CT were identified as independent predictors of confirmed COVID-19 in the multivariate analysis of the diagnostic cohort (Table 2).
In the univariate analysis of the cohort, the proportion of patients with at least 1 respiratory symptom among confirmed COVID-19 patients tended to be higher than that among non-confirmed COVID-19 patients $(P=$ 0.078). The other results are presented in Table 2.

Details of the infection control cohort: The characteristics of the 184 patients in the infection control cohort are shown in Table 3. Eleven patients were included in the high-risk group, and the remaining 173 patients were included in the intermediate-risk group before RT-LAMP (Fig. 3). Six (55\%) patients in the high-risk group and 5 patients $(2.9 \%)$ in the 
Table 3. The characteristics of the 184 cases in the infection control cohort

\begin{tabular}{|c|c|c|c|c|}
\hline \multirow[b]{2}{*}{ Characteristic } & \multicolumn{4}{|c|}{ Result for the following patients } \\
\hline & $\begin{array}{c}\text { Total } \\
(N=184)\end{array}$ & $\begin{array}{l}\text { COVID-19 cases } \\
\quad(N=11)\end{array}$ & $\begin{array}{l}\text { None COVID-19 cases } \\
\qquad(N=173)\end{array}$ & $P$ value \\
\hline Age, median years (range) & $72(23-97)$ & $78(37-91)$ & $71(23-97)$ & 0.37 \\
\hline \multicolumn{5}{|l|}{ Gender } \\
\hline Male / Female & $97 / 87$ & $4(36) / 7(64)$ & $93(54)$ / $80(46)$ & 0.35 \\
\hline \multicolumn{5}{|l|}{ Comobidities } \\
\hline Diabetes melitus & 31 & $2(18)$ & $29(17)$ & 1 \\
\hline Hypertension & 76 & $4(36)$ & $72(42)$ & 1 \\
\hline Chronic heart failure & 10 & $0(0)$ & $10(5.8)$ & 1 \\
\hline Chronic renal failure & 52 & $1(9.1)$ & $51(29)$ & 0.19 \\
\hline Chronic renal failure on hemodialysis & 8 & $1(9.1)$ & $7(4)$ & 0.4 \\
\hline Liver cirrosis & 3 & $0(0)$ & $3(1.7)$ & 1 \\
\hline Hematological malignancy & 29 & $1(9.1)$ & $28(16)$ & 1 \\
\hline Solid tumor & 45 & $0(0)$ & $45(26)$ & 0.68 \\
\hline Bronchial athma & 12 & $1(9.1)$ & $11(6.4)$ & 0.53 \\
\hline Chronic obstractive pulmonary disease & 7 & $0(0)$ & $7(4)$ & 1 \\
\hline Interstitial lung disease & 12 & $0(0)$ & $12(6.9)$ & 1 \\
\hline \multicolumn{5}{|l|}{ Medical status } \\
\hline Allgeneic hematopoietic stem cell transplantation & 11 & $0(0)$ & $11(6.4)$ & 1 \\
\hline Solid organ transplantation & 2 & $0(0)$ & $2(1.2)$ & 1 \\
\hline \multicolumn{5}{|l|}{ Clinical characteristics } \\
\hline Prior exposure to COVID-19 & 11 & $6(55)$ & $5(2.9)$ & 0.0000038 \\
\hline At least one respiratory symptom & 104 & $9(82)$ & $95(55)$ & 0.12 \\
\hline \multicolumn{5}{|l|}{ Chest CT imaging } \\
\hline Any bGGO on CT & 48 & $8(73)$ & $40(23)$ & 0.0011 \\
\hline bGGO without consolidation on $\mathrm{CT}$ & 36 & $6(55)$ & $30(17)$ & 0.0083 \\
\hline bGGO with consolidation(s) on CT & 12 & $2(18)$ & $10(5.8)$ & 0.15 \\
\hline
\end{tabular}

COVID-19, coronavirus disease 2019; bGGO, bilateral-ground-glass-opacity.

intermediate-risk group were diagnosed as confirmed COVID-19 patients. Of the patients in whom the results of the first RT-LAMP were negative (173 patients), 13 were categorized into the high-risk group by the assessment of ID experts (Fig. 3). The remaining 160 patients were categorized into the intermediate-risk group (Fig. 3). Although all 13 patients in the high-risk group underwent a second NAAT, none were positive. The duration of ICPs was significantly longer in the high-risk group (median 9 days, range 0-20 days) than among the intermediate-risk group (median 3 days, range $0-14$ days) $(P=0.00046)$. In addition, the duration of ICPs was significantly longer for the 40 patients with bGGO pneumonia (median 4 days, range 0-14 days) than for the remaining 133 patients (median 3 days, range $0-20$ days) $(P=0.0067)$.

Furthermore, 33 of the 160 patients included in the intermediate-risk group after the first RT-LAMP had pneumonia with bGGO on chest CT (Fig. 3). The duration of ICPs was significantly longer for the 33 patients with bGGO pneumonia (median, 4 days; range, 0-14 days) than for the remaining 127 patients (median, 3 days; range, $0-13$ days $)(P=0.005)$.

No clinical improvement was observed in 9 of these 160 patients. Thus, they underwent the second NAAT and were further assessed by ID experts; the second NAAT was negative in these 9 patients.
Analysis of possibility of nosocomial spread of SARS-CoV-2: No confirmed COVID-19 cases occurred in any of the hospitalized patients or staff at our institution during the study period or the subsequent 14-day observation period. In addition, there were no clusters of nosocomial respiratory infections, which would indicate possible COVID-19 transmission. However, there were 2 events in which some staff had contact with confirmed COVID-19 patients accidentally at the beginning of April before their definitive diagnosis. In total, 45 staff members had lowrisk exposure and 5 had intermediate-risk exposure. The 5 staff members who had intermediate-risk exposure were individually quarantined in their own homes for at least 14 days. Furthermore, respiratory infection (1 acute bronchitis and one pneumonia case) developed in 2 of the 50 staff members within 14 days of exposure. Although both of them underwent standard microbiological testing and 2 rounds of NAAT for SARS-CoV-2, the etiologies were uncertain.

\section{DISCUSSION}

The results of this study provide useful information about COVID-19 and insights into new infection control strategies (Figs. 1 and 2) based on the results of RT-LAMP, chest CT findings, and the clinical history of 
patients, which might aid efforts to protect all hospital staff and hospitalized patients from COVID-19 in the real-world clinical setting.

In this study, bGGO on chest CT and history of exposure to COVID-19 patients were identified as independent predictors of confirmed COVID-19 cases in the diagnostic cohort. Furthermore, infection control strategies (Figs. 1 and 2) were applied from the beginning of April 2020 with the aim of preventing the nosocomial spread of COVID-19 at our institution. Based on previous studies $(9,17,18)$, history of exposure and bGGO on chest CT were the cornerstones of our algorithms for assessing the risk of COVID-19 (Figs. 1 and 2). These 2 factors were also identified as independent predictors of COVID-19. Thus, the algorithms are reasonable in a real-world clinical setting.

The requirements for recommending discontinuation of the ICPs in the algorithm are shown in Fig. 2. False-negative NAAT results for SARS-CoV-2 have been reported; thus, ICPs should not be discontinued based on NAAT results alone $(8,19)$. Accordingly, we continued ICPs even when the results of RTLAMP were negative until the patients fulfilled the symptom-based requirements and/or were diagnosed with another illness (Fig. 2). In particular, high-risk patients with a history of exposure and intermediaterisk patients with pneumonia, seen as bGGO, required a significantly longer duration of ICPs in accordance with the algorithm (Fig. 2). It was thus probably rational to prevent the nosocomial spread of COVID-19 from the latent definite COVID-19 cases whose NAAT results were negative. In fact, there were no events that suggested nosocomial spread during the study period and the subsequent 14-day observation period carried out in accordance with the evidence-based standard infection control strategies $(1,13)$ and the algorithms, apart from the 2 patients suspected to have contracted the infection at the beginning of April when most staff were unaware of the algorithms. Although some nonemergency procedures were postponed, important medical care, such as allogeneic hematopoietic stem cell transplantation, surgery for cancer patients, and cancer chemotherapy were performed almost as usual even during that time (data not shown).

Furthermore, the algorithms could be utilized by using RT-PCR instead of RT-LAMP because the sensitivity and specificity of RT-LAMP for detecting SARS-CoV-2 were comparable to those of RT-PCR $(6,7)$. Therefore, our strategies may be applied at institutes and facilities globally as long as NAAT modalities, such as RT-PCR, are available. In addition, LAMP is simple and easy to perform and requires only a laboratory water bath on a heat block for the reactions (5). Thus, RT-LAMP might be useful, particularly in regions with limited medical resources, such as developing countries.

Our study has some limitations. First, this was a small retrospective study and not a randomized controlled trial, which was conducted to evaluate the utility of RT-LAMP-based algorithms and strategies. Thus, it is uncertain whether these algorithms and strategies are suitable for other clinical settings, including other hospitals and settings in Japan, where COVID-19 might be more prevalent than in our setting. Second, the false-negative rate of RT-LAMP has not yet been demonstrated. Other tests, such as serologic tests, should be performed to evaluate it. The false-negative rate of RT-PCR was estimated to range from $10 \%$ to $40 \%$ in a previous systematic review (19). During the study period, many patients with pneumonia were admitted to our hospital, some of whom probably had pneumonia associated with COVID-19, even though NAAT results were negative. In fact, there were 4 highrisk patients with respiratory infection who had a clear history of exposure to confirmed COVID-19 patients in the infection control cohort, although NAAT results were negative. These 4 patients were strongly suspected to have COVID-19. Third, quality control for RTLAMP was not performed using reference methods such as RT-PCR in this study. In addition, the same RNA extraction method for RT-LAMP has not been evaluated in previous studies, although the same amplification method (7) was used in the present study. Therefore, the sensitivity of RT-LAMP in this study might be lower than that reported in previous studies $(6,7)$. As a result, the 2 staff members who had respiratory infections within 14 days after exposure might not have been diagnosed with confirmed COVID-19 patients by RTLAMP in this study.

In conclusion, we have reported infection control strategies based on RT-LAMP. Establishing a strong comprehensive system that combines new strategies based on NAAT modalities with evidence-based standard infection control strategies will potentially be useful to prevent the nosocomial spread of SARSCoV-2 in the era of COVID-19.

Acknowledgments We thank Ms. Yukiko Fuke, Ms. Hanae Uno, Dr. Sara Ikeda, Dr. Akiko Yoneyama, and the staff of the Microbiology Laboratory at Toranomon Hospital for supporting the infection control strategies.

Conflict of interest None to declare.

\section{REFERENCES}

1. Lynch JB, Davitkov P, Anderson DJ, et al. Infectious Diseases Society of America Guidelines on Infection Prevention for Health Care Personnel Caring for Patients with Suspected or Known COVID-19. Available at <https://www.idsociety.org/globalassets/ idsa/practice-guidelines/covid-19/infection-prevention/idsacovid-19-guideline_ip_v1.0.1.pdf > . Accessed October 31, 2020.

2. Stokes EK, Zambrano LD, Anderson KN, et al. Coronavirus disease 2019 case surveillance - United States, January 22-May 30, 2020. MMWR Morb Mortal Wkly Rep. 2020; 69:759-65.

3. Hanson KE, Caliendo AM, Arias CA, et al. Infectious Diseases Society of America Guidelines on the Diagnosis of COVID-19. Available at $<$ https://www.idsociety.org/globalassets/idsa/ practice-guidelines/covid-19/diagnostics/idsa-covid-19guideline_dx_version-1.0.1.pdf $>$. Accessed October 31, 2020.

4. World Health Organization (WHO). Laboratory testing for coronavirus disease (COVID-19) in suspected human cases: interim guidance. 19 March 2020. Available at <https://www. who.int/publications/i/item/laboratory-testing-for-2019-novelcoronavirus-in-suspected-human-cases-20200117>. Accessed October 31, 2020.

5. Notomi T, Okayama H, Masubuchi H, et al. Loop-mediated isothermal amplification of DNA. Nucleic Acids Res. 2000; 28: E63.

6. Yan C, Cui J, Huang L, et al. Rapid and visual detection of 2019 novel coronavirus (SARS-CoV-2) by a reverse transcription loopmediated isothermal amplification assay. Clin Microbiol Infect. 
2020; 26:773-9

7. Kitagawa Y, Orihara Y, Kawamura R, et al. Evaluation of rapid diagnosis of novel coronavirus disease (COVID-19) using loop-mediated isothermal amplification. J Clin Virol. 2020; 129:104446.

8. Kucirka LM, Lauer SA, Laeyendecker O, et al. Variation in falsenegative rate of reverse transcriptase polymerase chain reactionbased SARS-CoV-2 tests by time since exposure. Ann Intern Med. 2020; 173:262-7.

9. Centers for Disease Control and Prevention (CDC). Discontinuation of Transmission-Based Precautions and Disposition of Patients with COVID-19 in Healthcare Settings (Interim Guidance). Available at $<$ https://www.cdc.gov/ coronavirus/2019-ncov/hcp/disposition-hospitalized-patients. html >. Accessed October 31, 2020.

10. Bi Q, Wu Y, Mei S, et al. Epidemiology and transmission of COVID-19 in 391 cases and 1286 of their close contacts in Shenzhen, China: a retrospective cohort study. Lancet Infect Dis. 2020; 20:911-9.

11. Japanese Society for Infection Prevention and Control. Guidance on implementations for COVID-19 in healthcare facilities. The third edition. Available at <http://www.kankyokansen. org/uploads/uploads/files/jsipc/COVID-19_taioguide3.pdf $>$. Accessed October 31, 2020. Japanese.

12. CDC. Interim U.S. Guidance for Risk Assessment and Work Restrictions for Healthcare Personnel with Potential Exposure to COVID-19. Updated Oct 21, 2020. Available at <https://www. cdc.gov/coronavirus/2019-ncov/hcp/guidance-risk-assesmenthcp.html>. Accessed October 31, 2020.

13. WHO. Infection prevention and control during health care when coronavirus disease (COVID-19) is suspected or confirmed. Interim guidance 29 June 2020. Available at $<$ https:/www.who. int/publications/i/item/WHO-2019-nCoV-IPC-2020.4>. Accessed October 31, 2020.

14. WHO. WHO Guidelines on Hand Hygiene in Health Care Available at $<$ https://apps.who.int/iris/bitstream/hand le/10665/44102/9789241597906_eng.pdf;jsessionid=8EBD6 60419F3CF020E36B6D18A2835B6? sequence $=1>$. Accessed October 31, 2020

15. Corman VM, Landt O, Kaiser M, et al. Detection of 2019 novel coronavirus (2019-nCoV) by real-time RT-PCR. Euro Surveill. 2020; 25:2000045.

16. Kanda Y. Investigation of the freely available easy-to-use software 'EZR' for medical statistics. Bone Marrow Transplant. 2013; 48:452-8.

17. Zhao W, Zhong Z, Xie X et al. Relation between chest $\mathrm{CT}$ findings and clinical conditions of coronavirus disease (COVID-19) pneumonia: a multicenter study. Am J Roentgenol. 2020; 214:1072-7.

18. Shi H, Han X, Jiang N, et al. Radiological findings from 81 patients with COVID-19 pneumonia in Wuhan, China: a descriptive study. Lancet Infect Dis. 2020; 20:425-34.

19. Weissleder R, Lee H, Ko J, et al. COVID-19 diagnostics in context. Sci Transl Med. 2020; 12:eabc1931. 